Check for updates

Cite this: Chem. Sci., 2019, 10, 3969

๑ All publication charges for this article have been paid for by the Royal Society of Chemistry

Received 21st August 2018

Accepted 20th February 2019

DOI: $10.1039 / \mathrm{c} 8 \mathrm{sc} 03725 f$

rsc.li/chemical-science

\section{Annihilator dimers enhance triplet fusion upconversion $\uparrow$}

\author{
Andrew B. Pun, (D) a Samuel N. Sanders, ${ }^{b}$ Matthew Y. Sfeir, (D) ${ }^{\text {cd }}$ Luis M. Campos ${ }^{\star a}$ \\ and Daniel N. Congreve (D) *b
}

\begin{abstract}
Optical upconversion is a net process by which two low energy photons are converted into one higher energy photon. There is vast potential to exploit upconversion in applications ranging from solar energy and biological imaging to data storage and photocatalysis. Here, we link two upconverting chromophores together to synthesize a series of novel tetracene dimers for use as annihilators. When compared with the monomer annihilator, TIPS-tetracene, the dimers yield a strong enhancement in the triplet fusion process, also known as triplet-triplet annihilation, as demonstrated via a large increase in upconversion efficiency and an order of magnitude reduction of the threshold power for maximum yield. Along with the ongoing rapid improvements to sensitizer materials, the dimerization improvements demonstrated here open the way to a wide variety of emerging upconversion applications.
\end{abstract}

\section{Introduction}

The ability to design and synthesize materials that convert infrared light into visible light is remarkably important due to widespread potential applications, including solar energy capture, ${ }^{1}$ photocatalysis, ${ }^{2}$ data storage,${ }^{3}$ night vision, ${ }^{4}$ and biological imaging. ${ }^{5}$ In this vein, the process by which low energy photons are converted to a single high energy photon is known as optical upconversion. ${ }^{6,7}$ In recent years this process has received a surge of interest for its potential in solar energy applications. ${ }^{8}$ As a result, much work has been done to optimize upconversion in highly ordered and condensed systems, where there are large numbers of annihilators in very close proximity, thus leading to high upconversion efficiencies. ${ }^{9}$ Considering that upconversion can also greatly benefit other areas, such as imaging $^{10}$ and photochemistry, ${ }^{2,11}$ where lower concentrations are required, a major challenge remains to find efficient upconverting materials that are robust with respect to concentration level. Thus, it is imperative to investigate how structural modifications to annihilators can be exploited to tune the process of upconversion.

\footnotetext{
${ }^{a}$ Department of Chemistry, Columbia University, New York, New York 10027, USA. E-mail: lcampos@columbia.edu

${ }^{b}$ Rowland Institute at Harvard University, Cambridge, Massachusetts 02142, USA. E-mail: congreve@rowland.harvard.edu

'Photonics Initiative, Advanced Science Research Center, City University of New York, New York, New York 10031, USA

${ }^{d}$ Department of Physics, Graduate Center, City University of New York, New York, New York 10016, USA

$\dagger$ Electronic supplementary information (ESI) available. See DOI: $10.1039 / \mathrm{c} 8 \mathrm{sc} 03725 \mathrm{f}$
}

One promising approach to exhibit efficient upconversion is using mixed conjugated organic systems operating via triplet fusion (TF) upconversion, also known as triplet-triplet annihilation upconversion (Fig. 1A). ${ }^{12,13}$ In such systems, an organometallic sensitizer absorbs long wavelength light, exciting the sensitizer to a singlet state, which then rapidly undergoes intersystem crossing to a long lived triplet state, shown in Fig. 1B. This triplet can then be transferred from the sensitizer to an organic annihilator via collisional triplet energy transfer (TET), provided the triplet energy level of the annihilator is lower than that of the sensitizer. Two annihilators in their excited triplet states $\left(T_{1}\right)$ can then come together and undergo intermolecular triplet fusion (xTF), populating the excited singlet $\left(\mathrm{S}_{1}\right)$ state of one of the annihilators. This $\mathrm{S}_{1}$ then decays to the ground state singlet $\left(\mathrm{S}_{0}\right)$ via fluorescence, giving off a photon of higher energy than the incident light. In order for the xTF process to occur, it must conserve energy, such that the energy of the singlet state is less than twice the energy of the triplet state, i.e. $E\left(\mathrm{~S}_{1}\right)<2 \times E\left(\mathrm{~T}_{1}\right)$. A schematic of the xTF process can be seen in Fig. 1C.

In order for TF to be a technically viable process, it is necessary to absorb low energy photons (such as IR and near infrared, NIR) that are inaccessible to many optoelectronic materials. Thus, a major focus in the field of TF upconversion has been on finding sensitizers which can absorb sufficiently low energy. Great strides have been made in recent years to this end, including the use of lead and cadmium chalcogenide nanocrystals as sensitizers to complement existing molecular annihilators. ${ }^{14-17}$ While much focus has been given to improving the sensitizer, comparatively little work has been done on the annihilator. ${ }^{18,19}$ Novel and exciting methods focusing on the structure of the materials to assemble the 
A

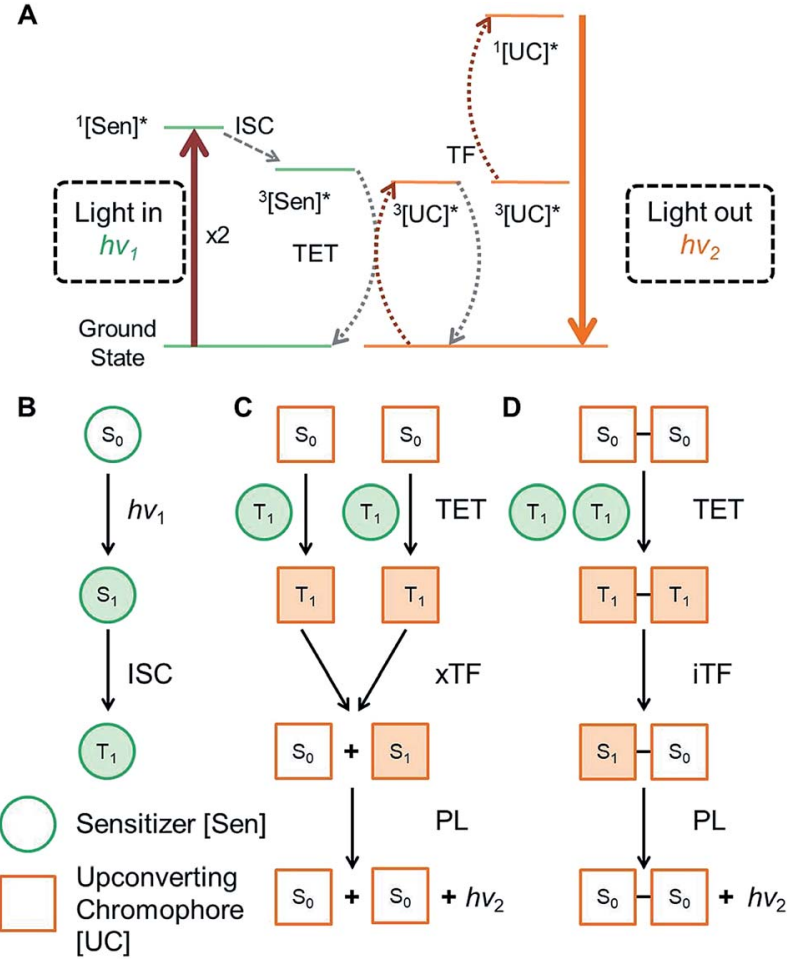

Fig. 1 (A) Qualitative energy level diagram of the full triplet fusion upconversion mechanism. *Denotes first excited state ([Sen] = sensitizer, $[U C]=$ upconverting chromophore) (B) mechanism by which triplet states are populated in a molecular sensitizer by photoexcitation $\left(h \nu_{1}\right)$, followed by intersystem crossing (ISC). (C) Mechanism of conventional intermolecular triplet fusion upconversion (xTF) giving off a high energy photon $\left(h \nu_{2}\right)$. (D) Proposed mechanism for intramolecular triplet fusion upconversion (iTF). Unfilled and filled shapes represent ground state and excited state molecules, respectively. $\mathrm{S}$ and $T$ denote singlet and triplet states respectively.

annihilators have been explored by the Simon and Kimizuka groups. ${ }^{20-22}$ But to date, there has been very little work to modify the annihilator itself to intrinsically enhance upconversion, especially operating at low concentrations in solution. Thus far, most work in the field of optical upconversion has employed common acene derivatives, such as diphenyl anthracene and rubrene, or perylene as the organic upconverter. ${ }^{23-25}$ In this work, we investigate how covalently coupling two upconverting chromophores to form an annihilator dimer affects the efficiency of upconversion. In contrast to previously studied polymeric systems, which focus on large assemblies, ${ }^{26-28}$ we sought to investigate interactions between two individual chromophores. Thus, we synthesized a series of tetracene dimers linked by $0,1,2$, and $4 p$-phenylene spacers $(\mathrm{BT} n ; n=0,1,2,4)$, which were designed to undergo intramolecular triplet fusion upconversion (iTF, Fig. 1D), in addition to the typical xTF caused by collisions between chromophores.

\section{Experimental}

\section{Synthesis of BT0,1,2 and 4}

Full synthetic details for BT0, BT1, BT2 and BT4 including characterization data is provided in the ESI. $\dagger$

\section{Optical characterization}

Solutions of BT0,1,2,4 or TIPS-tetracene (TIPS-Tc) with $\mathrm{PdPc}(\mathrm{OBu})_{8}$ were prepared from anhydrous toluene in a nitrogen glovebox. Solutions were made in $1 \mathrm{~cm} \times 1 \mathrm{~cm}$ cuvettes from Spectrocell and were degassed by sparging with nitrogen for 30 seconds then sealed before removing from glovebox for measurement. Solutions were excited with a $730 \mathrm{~nm}$ laser diode purchased from Thorlabs, focused to a beam diameter of 0.15 $\mathrm{mm}$. Unless otherwise noted, the excitation density in all experiments was $113 \mathrm{~W} \mathrm{~cm}^{-2}$. All upconverted PL spectra were measured with a QEPro spectrometer purchased from Ocean Optics through a $700 \mathrm{~nm}$ shortpass filter. Power intensity dependence measurements were taken by varying the beam intensity using neutral density filters.

\section{PLQY}

PLQY measurements were made using an integrating sphere purchased from Labsphere following de Mello et al. ${ }^{29}$ Briefly, the $10 \mathrm{~mm}$ cuvette was placed inside the sphere and excited with either $730 \mathrm{~nm}$ or $445 \mathrm{~nm}$ light focused from a laser diode. The upconversion quantum yield was determined by comparing the quantum yields when excited at $730 \mathrm{~nm}$ and $445 \mathrm{~nm}$, with the upconversion yield defined such that it is the number of excitations leading to a singlet exciton on the annihilator. The cited yields are for annihilator concentrations of $5 \times 10^{-4} \mathrm{M}$. The sphere and all optical equipment were calibrated against a calibrated silicon photodetector from Newport Corp.

\section{Results and discussion}

Our approach in designing annihilators for upconversion is rooted in our fundamental understanding of how the platform of acene dimers operates in intramolecular singlet fission (iSF) the reverse process of iTF. ${ }^{30}$ In all reported acene dimers, the spatial proximity of the generated triplet pairs leads to extremely rapid recombination. ${ }^{31}$ Even in the case of acene chromophores spaced by up to two $p$-phenylene spacers, the lifetime of a pair of triplets on one acene dimer is no more than $270 \mathrm{~ns},{ }^{30,32}$ ensuring us that iTF can occur with a relatively long bridge between the chromophores. However, it should be noted that when the energetic criteria for iSF is not met, the dimeric system is ideal to undergo iTF, because of the aforementioned rapid triplet recombination. Considering that the energy of the singlet state in tetracene is roughly less than twice the energy of the triplet state - an observation that is not favorable for iSF but good for iTF - we investigate the upconversion process in a series of tetracene dimers shown in Fig. 2A. The tetracene dimer BT0 does not contain a bridge, but BT1 and BT2 contain a phenylene and biphenylene bridge, respectively. These bridges effectively separate the triplets after sensitization, but maintain electronic communication between them. We also synthesized a tetracene dimer with a quaterphenylene bridge, BT4, to observe the effects of dimerization in a system where the two tetracene chromophores are too distant to be in good electronic communication. BT4 was chosen as it is the longest $p$-phenylene spaced dimer we could readily synthesize. These compounds were chosen due to 
A<smiles>[R7]c1c2ccccc2c([R7])c2cc3ccccc3cc12</smiles>

TIPS-TC

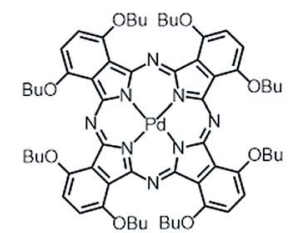

$\mathrm{PdPc}(\mathrm{OBu})_{8}$
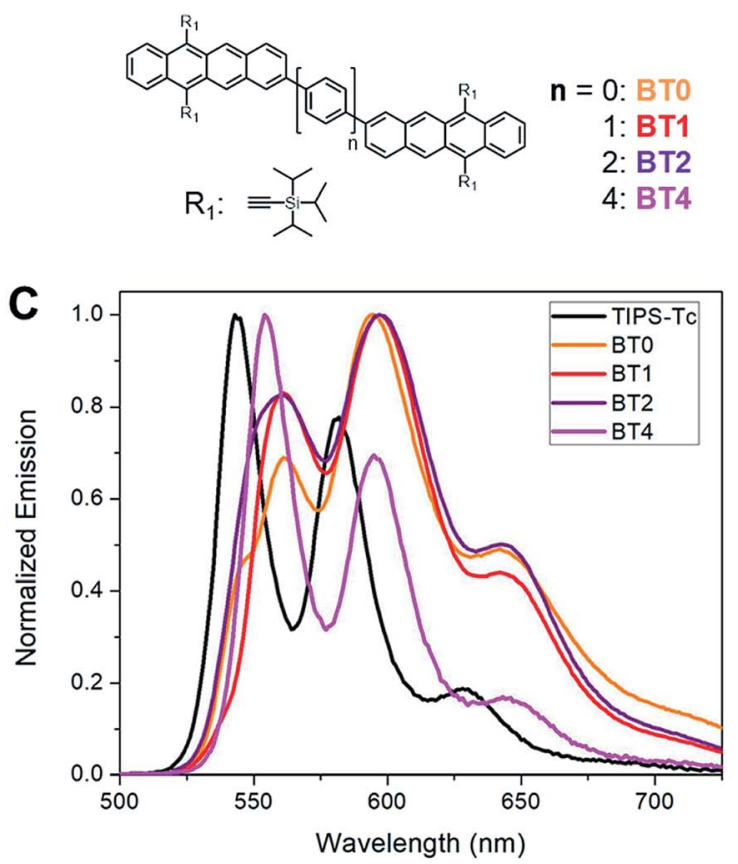

B
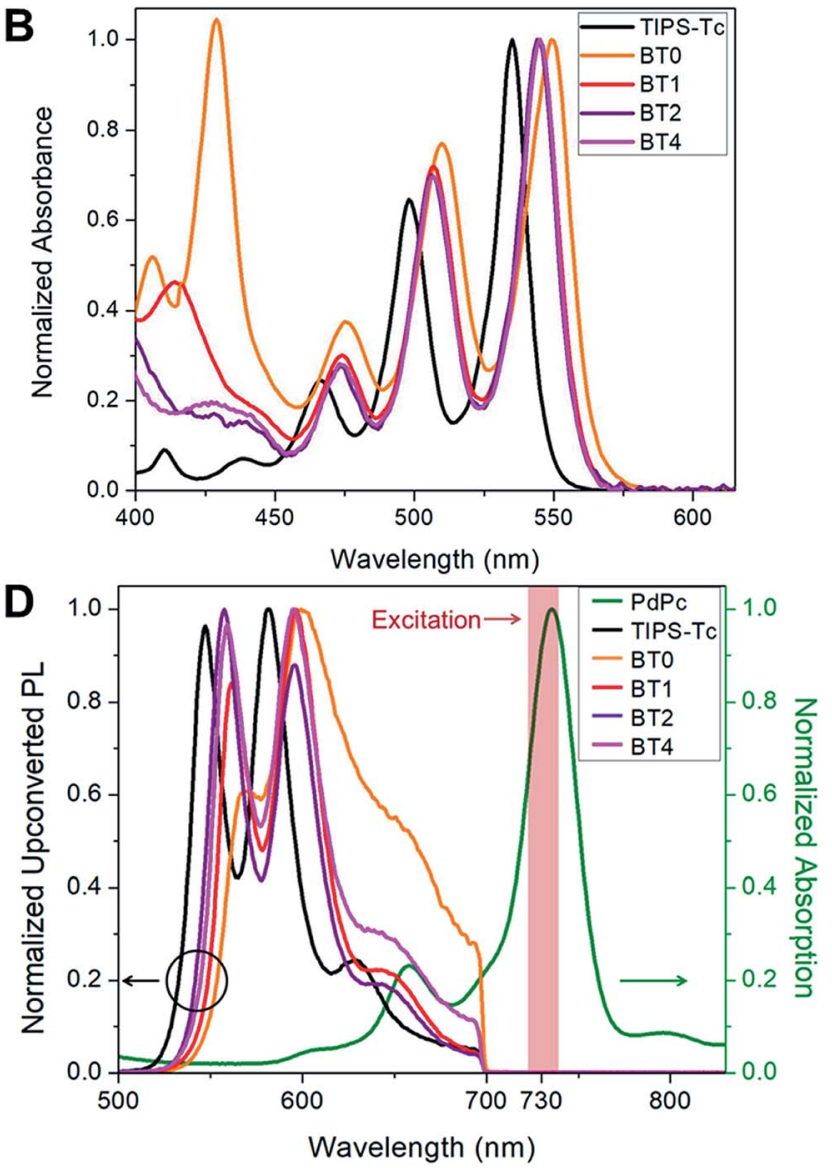

Fig. 2 (A) Structures of the compounds used in this work. (B) Normalized steady state absorbance spectra of the annihilators used. (C) Normalized photoluminescence spectra of the annihilators used, excited at $490 \mathrm{~nm}$. (D) Normalized upconverted photoluminescence spectra at $5 \times 10^{-4} \mathrm{M}$ annihilator and $2.5 \times 10^{-5} \mathrm{M} \mathrm{PdPc}(\mathrm{OBu})_{8}\left(700 \mathrm{~nm}\right.$ short pass filter used). Normalized steady state absorbance of $\mathrm{PdPc}(\mathrm{OBu})_{8}$ shown in green. The shaded red region represents excitation wavelength from $730 \mathrm{~nm}$ diode.

the presence of the triisopropylsilylacetylene (TIPS) groups which impart stability and solubility, as well as facilitating iterative synthesis. ${ }^{33}$ These compounds were compared to a monomeric TIPS-Tc annihilator. $\mathrm{PdPc}(\mathrm{OBu})_{8}$ was chosen as the sensitizer, as it has been previously shown that this compound can efficiently donate triplets to tetracene derivatives upon absorption of near IR light. ${ }^{34}$

The absorption and emission spectra of the annihilators used in this work can be seen in Fig. 2B and C, respectively. The absorption of the tetracene dimers exhibits a modest redshift in the onset of absorption relative to TIPS-Tc. This is characteristic of the extension of a $\pi$ system typically seen in conjugated organic molecules. The emission spectra of the upconverting chromophores are similar to one another, with TIPS-Tc and BT4 exhibiting a more pronounced peak at $550 \mathrm{~nm}$ relative to the peak at $600 \mathrm{~nm}$, and vice versa for BT0-BT2. The normalized absorption spectra of $\mathrm{PdPc}(\mathrm{OBu})_{8}$ can be seen in green in Fig. 2D, exhibiting strong absorption in the near IR. Upon excitation with a $730 \mathrm{~nm}$ laser diode (shaded red Fig. 2D), optical upconversion was observed for TIPS-Tc as well as all four tetracene dimers. The normalized upconversion photoluminescence (UCPL) of these compounds can be seen in Fig. 2D.
To confirm that iTF was possible, we performed DFT calculations of the $\mathrm{T}_{1}$ states of BT0, BT1, BT2, and BT4.$^{35}$ Fig. 3 shows one of the doubly degenerate $\mathrm{T}_{1}$ orbitals of BT0, BT1, BT2, and BT4. In the case of closely linked dimers such as BT0, BT1, and BT2, the $T_{1}$ state is generally localized on one of the tetracene chromophores, but spills onto the second chromophore. This mixing of triplet states across multiple chromophores facilitates iTF. In the case of BT4, the two tetracene chromophores are too far apart to allow the $T_{1}$ state to be shared. As a result, BT4 should not be able to undergo iTF, and instead we would expect it to behave similarly to TIPS-Tc as an upconversion annihilator.

After establishing the presence of upconversion when using tetracene dimers as annihilators, we explored the dependence of upconversion photoluminescence intensity on the concentration of these materials. We began with a sensitizer concentration of $2.5 \times 10^{-5} \mathrm{M}$, and an annihilator concentration of $5 \times$ $10^{-4} \mathrm{M}$, these being typical of solution state measurements of upconversion. ${ }^{19,34}$ We then lowered the concentration of the annihilator down to $3.75 \times 10^{-5} \mathrm{M}$ while holding the amount of sensitizer constant in order to study the effects of iTF on concentration. The dependence of upconversion PL as 

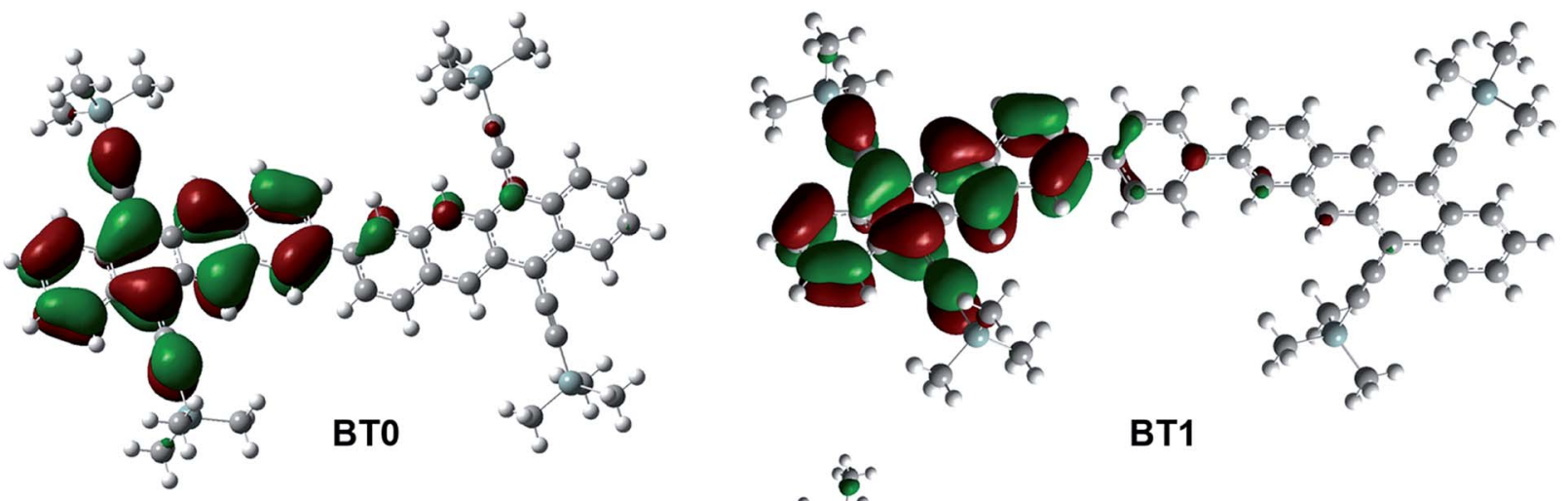

BT1

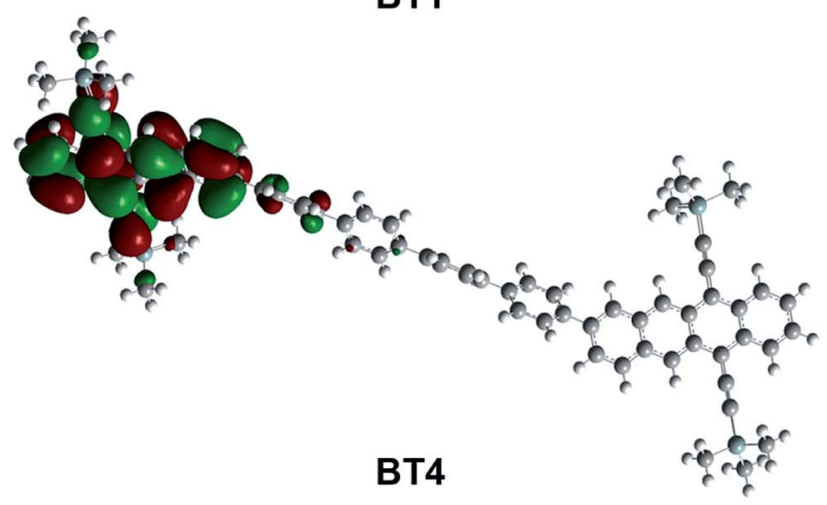

Fig. $3 \mathrm{~T}_{1}$ orbitals of tetracene dimers studied generated via DFT calculations.

a function of concentration of the annihilator can be seen in Fig. 4A. BT4 decays nearly identically to the monomeric TIPSTc, as we would expect in a system where the two tetracene chromophores are electronically independent of one another and lack the iTF upconversion pathway. BT0 exhibits the lowest upconversion PL across all concentrations, which is likely due to its low photoluminescence quantum yield (PLQY) as a result of singlet fission, a competing pathway for singlet decay. ${ }^{36,37} \mathrm{In}$ stark contrast, BT1 and BT2 both exhibit higher upconversion PL compared to TIPS-Tc, across all concentrations. This is even in spite of the significantly lower PLQYs of BT1 and BT2, a result of the fact that these compounds undergo singlet fission as well. In fact, the overall upconversion yield (defined as the percentage of absorbed photons that become a singlet on the annihilator) is significantly higher in BT1 than in TIPS-Tc, at $4.2 \%$ vs. $0.70 \%$, respectively. This upconversion yield is equivalent to that of a previously reported tetracene dimer ${ }^{19}$ but we note that we are using a phosphor that absorbs lower energy light and lower concentrations of sensitizer. Calculations show that the $T_{1}$ energies of the annihilators studied in this work vary by only $7 \mathrm{meV}$ (Table S1†), ruling out changes in $\mathrm{T}_{1}$ energy as the source of our enhanced upconversion performance in these materials. Indeed, the robustness of the BT1 upconverting chromophore to these non-ideal conditions proves its usefulness in a wide variety of demanding upconversion applications where lower concentrations are required.

We then performed Stern-Volmer quenching experiments on our system to measure the rate of triplet energy transfer $\left(k_{\mathrm{TET}}\right)$ from sensitizer to annihilator. This was done to rule out an increase in $k_{\mathrm{TET}}$ as the source of enhanced UCPL in our tetracene dimers (Fig. S9, Table S2 $\dagger$ ). These measurements showed a slight enhancement in $k_{\mathrm{TET}}$ of the dimers relative to TIPS-Tc. The change in $k_{\mathrm{TET}}$ is small relative to the overall enhancement in UCPL for these materials, but in order to provide further evidence of iTF, we constructed a kinetic model of our system. The details of this model can be found in the ESI (Fig. S11 $\dagger$ ). Using this model, we are able to fit the rate of triplet fusion $\left(k_{\mathrm{TF}}\right)$ to our experimental data in Fig. 4A. Using the values of $k_{\text {TET }}$ extracted from our Stern-Volmer measurements, we find the only way to fit our data using the model is with an enhanced rate of $k_{\mathrm{TF}}$ (Table S6 $\dagger$ ). TIPS-Tc has a $k_{\mathrm{TF}}$ of $2.3 \times 10^{6}\left(\mathrm{M}^{-1} \mathrm{~s}^{-1}\right)$, compared to $250 \times 10^{6}\left(\mathrm{M}^{-1} \mathrm{~s}^{-1}\right)$ for BT1. We believe this enhancement in $k_{\mathrm{TF}}$ is additional supporting evidence of iTF, which leads to enhanced UPCL yields of our BT dimers.

In the case of classical xTF, a high concentration of annihilator molecules has been considered crucial for efficient upconversion, despite the fact that this high concentration can lead to fluorescence quenching via aggregation. ${ }^{7,38,39}$ At high annihilator concentrations, we expect xTF to dominate (that is, excited annihilator molecules are much more likely to collide with one another than with a sensitizer). This is demonstrated by the fact that TIPS-Tc is competitive with both BT1 and BT2 at higher concentrations. At low concentrations, however, excited annihilators are as likely to collide with sensitizers as they are with another annihilator, and thus the ability to hold two triplets is greatly advantageous, and the dimeric materials dominate, suggesting that iTF is occurring. This is exhibited by the greater than an order of magnitude reduction of UCPL for TIPS-Tc, while BT1 and BT2 only see a modest decrease in UCPL at low concentrations (Fig. 4A). For this reason, most demonstrations of 

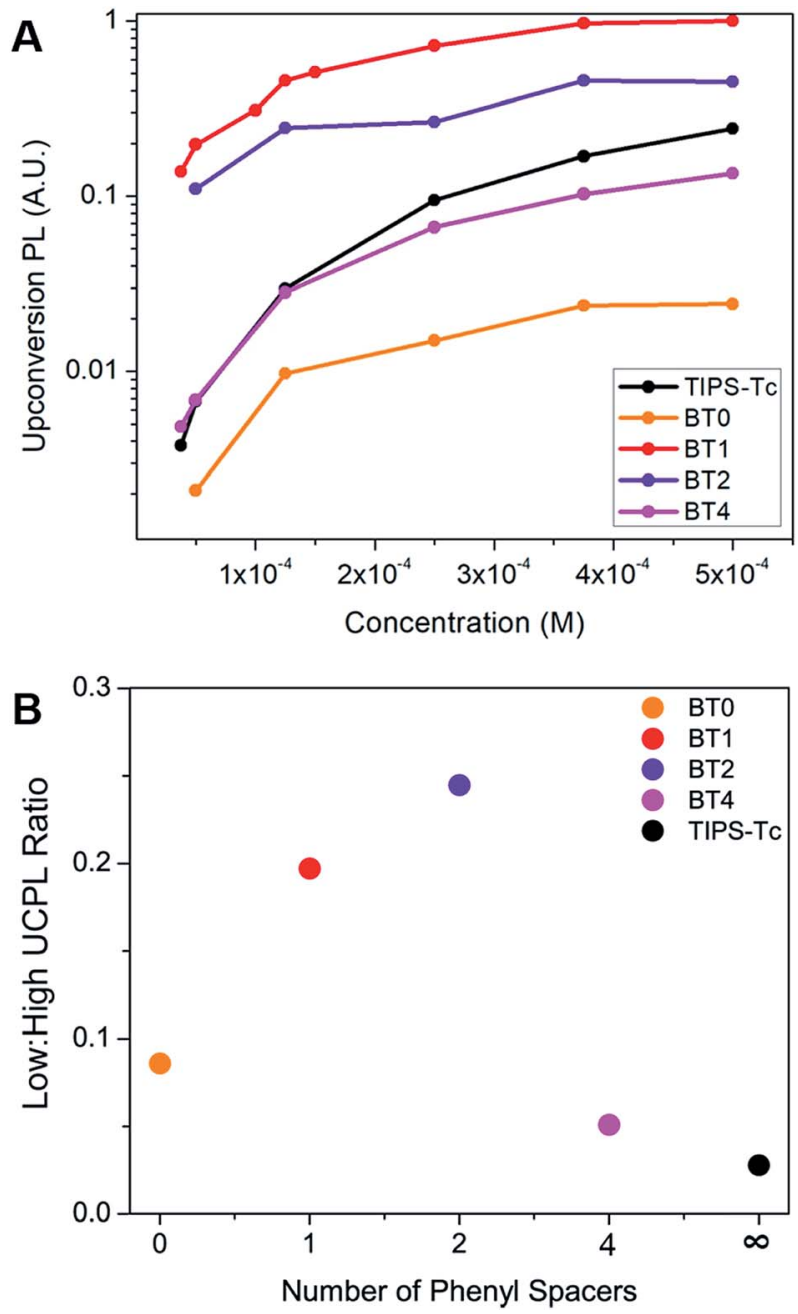

Fig. 4 (A) Change in upconversion PL of compounds studied as a function of annihilator concentration at $2.5 \times 10^{-5} \mathrm{M} \mathrm{PdPc}(\mathrm{OBu})_{8}$. (B) UCPL at $5 \times 10^{-4} \mathrm{M}$ divided by UCPL at $5 \times 10^{-5} \mathrm{M}$ annihilator concentration plotted as a function of the number of phenyl spacers in the annihilator used.

upconversion in solution do so with an annihilator concentration of $\sim 1 \mathrm{mM}$, whereas we can see bright upconversion even at annihilator concentrations as low as $3.75 \times 10^{-5} \mathrm{M}$. The benefit of the extra iTF pathway can clearly be seen in its high upconversion PL seen at very low concentrations. This is further demonstrated in Fig. 4B, where we compare the UCPL of these annihilators and low and high concentration. This opens the door for the use of iTF capable upconversion materials in photochemical or imaging applications, where high concentrations of extrinsic materials are undesirable. For example, it is known that a high concentration of annihilators will reduce the propagation of the upconverted PL in solution. ${ }^{40}$ This is disadvantageous for imaging applications where it is crucial that the upconverted PL be detectable throughout the species. Photochemical reactions where the photocatalyst is excited by upconverted $\mathrm{PL}^{\mathbf{1 1}}$ would also benefit from a low concentration of annihilator, because a high concentration of these extra species could easily lead to undesirable side reactions.

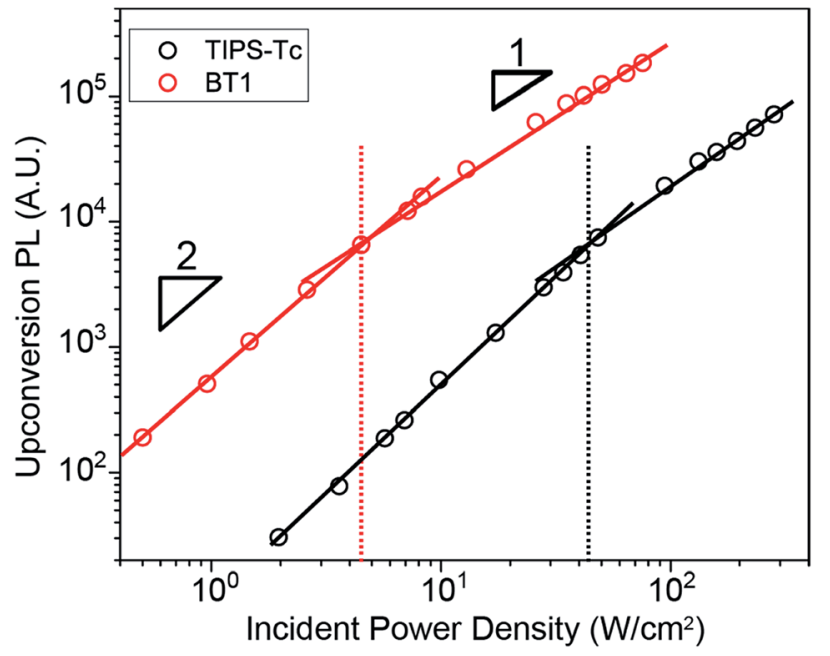

Fig. 5 Dependence of upconverted PL on incident light intensity at 2.5 $\times 10^{-4} \mathrm{M}$ TIPS-TC and BT1 concentration. The transition between quadratic and linear dependences occurs at 44.5 and $4.3 \mathrm{~W} \mathrm{~cm}^{-2}$ for TIPS-TC and BT1 respectively.

Because it requires two initial species, upconversion PL has a quadratic dependence on light intensity at low fluence. This dependence on light intensity then exhibits a change from quadratic to linear once TF becomes the dominant recombination mechanism. ${ }^{41}$ Fig. 5 shows the dependence of upconversion PL intensity on incident power density for TIPS-Tc and BT1 (BT0, BT2, and BT4 shown in Fig. S1, S2, and S3† respectively). The crossover point of BT1, $4.3 \mathrm{~W} \mathrm{~cm}^{-2}$ is an order of magnitude lower than that of TIPS-Tc at $44.5 \mathrm{~W} \mathrm{~cm}^{-2}$. This crossover point reduction is a crucial benefit for BT1, as it ensures that upconversion is occurring at maximum efficiency even at low photon flux.

\section{Conclusions}

In conclusion, we have synthesized a new series of tetracene dimers that suggest that intramolecular triplet fusion is occurring in these materials. Dimerization is an attractive strategy towards enhanced upconversion because it offers intrinsic benefits to upconversion, rather than relying on complex processing or assembly techniques. This should ease the transition of these materials into non-ideal media where they could be widely applicable, such as in biological systems or photochemical reactions, where a greater number of exogenous species can reduce the efficiency of xTF. As compared to monomeric TIPS-Tc, a tetracene dimer exhibits greater upconversion yield, less sensitivity to concentration, and reduced power thresholds. These improvements pave the way towards upconversion as a viable candidate for a host of applications, especially when a high concentration of outside species is either unfavorable or untenable.

\section{Conflicts of interest}

The authors declare the following competing financial interest(s): Columbia University is filing a patent based on this work. 


\section{Acknowledgements}

A. B. P. thanks the NSF for GRFP (DGE 16-44869). L. M. C. acknowledges support from the Cottrell Scholar Award and the National Science Foundation (NSF) CAREER Program (DMR 1351293). We are grateful to Jonathan Owen for use of the fluorimeter. D. N. C. acknowledges the support of the Rowland Fellowship Program of the Rowland Institute at Harvard University. This work used the Extreme Science and Engineering Discovery Environment (XSEDE), which is supported by National Science Foundation grant number TG-DMR150018. This research used resources of the Center for Functional Nanomaterials, which is a US DOE Office of Science Facility at Brookhaven National Laboratory under contract No. DESC0012704.

\section{Notes and references}

1 T. F. Schulze and T. W. Schmidt, Energy Environ. Sci., 2015, 8, 103-125.

2 B. D. Ravetz, A. B. Pun, E. M. Churchill, D. N. Congreve, T. Rovis and L. M. Campos, Nature, 2019, 565, 343-346.

3 Y. Zhou, S.-T. Han, X. Chen, F. Wang, Y.-B. Tang and V. A. L. Roy, Nat. Commun., 2014, 5, 4720.

4 S.-W. Liu, C.-C. Lee, C.-H. Yuan, W.-C. Su, S.-Y. Lin, W.-C. Chang, B.-Y. Huang, C.-F. Lin, Y.-Z. Lee, T.-H. Su and K.-T. Chen, Adv. Mater., 2015, 27, 1217-1222.

5 Y. Il Park, K. T. Lee, Y. D. Suh and T. Hyeon, Chem. Soc. Rev., 2015, 44, 1302-1317.

6 T. W. Schmidt and F. N. Castellano, J. Phys. Chem. Lett., 2014, 5, 4062-4072.

7 J. Zhou, Q. Liu, W. Feng, Y. Sun and F. Li, Chem. Rev., 2015, 115, 395-465.

8 J. C. Goldschmidt and S. Fischer, Adv. Opt. Mater., 2015, 3, 510-535.

9 P. Bharmoria, S. Hisamitsu, H. Nagatomi, T. Ogawa, M. Morikawa, N. Yanai and N. Kimizuka, J. Am. Chem. Soc., 2018, 140, 10848-10855.

10 U. Agrawal, D. T. Reilly and C. M. Schroeder, Curr. Opin. Biotechnol., 2013, 24, 646-653.

11 S. H. C. Askes, A. Bahreman and S. Bonnet, Angew. Chem., Int. Ed., 2014, 53, 1029-1033.

12 T. N. Singh-Rachford and F. N. Castellano, Coord. Chem. Rev., 2010, 254, 2560-2573.

13 S. Baluschev, T. Miteva, V. Yakutkin, G. Nelles, A. Yasuda and G. Wegner, Phys. Rev. Lett., 2006, 97, 143903.

14 M. Wu, D. N. Congreve, M. W. B. Wilson, J. Jean, N. Geva, M. Welborn, T. Van Voorhis, V. Bulović, M. G. Bawendi and M. A. Baldo, Nat. Photonics, 2016, 10, 31-34.

15 Z. Huang, X. Li, M. Mahboub, K. M. Hanson, V. M. Nichols, H. Le, M. L. Tang and C. J. Bardeen, Nano Lett., 2015, 15, 5552-5557.

16 Z. Huang, X. Li, B. D. Yip, J. M. Rubalcava, C. J. Bardeen and M. L. Tang, Chem. Mater., 2015, 27, 7503-7507.

17 V. Gray, P. Xia, Z. Huang, E. Moses, A. Fast, D. A. Fishman, V. I. Vullev, M. Abrahamsson, K. Moth-Poulsen and M. Lee Tang, Chem. Sci., 2017, 8, 5488-5496.
18 D. Dzebo, K. Börjesson, V. Gray, K. Moth-Poulsen and B. Albinsson, J. Phys. Chem. C, 2016, 120, 23397-23406.

19 S. Baluschev, V. Yakutkin, T. Miteva, Y. Avlasevich, S. Chernov, S. Aleshchenkov, G. Nelles, A. Cheprakov, A. Yasuda, K. Müllen and G. Wegner, Angew. Chem., Int. Ed., 2007, 46, 7693-7696.

20 D. C. Thévenaz, S. H. Lee, F. Guignard, S. Balog, M. Lattuada, C. Weder and Y. C. Simon, Macromol. Rapid Commun., 2016, 37, 826-832.

21 P. Duan, N. Yanai, H. Nagatomi and N. Kimizuka, J. Am. Chem. Soc., 2015, 137, 1887-1894.

22 K. Mase, K. Okumura, N. Yanai and N. Kimizuka, Chem. Commun., 2017, 53, 8261-8264.

23 D. Di, L. Yang, J. M. Richter, L. Meraldi, R. M. Altamimi, A. Y. Alyamani, D. Credgington, K. P. Musselman, J. L. MacManus-Driscoll and R. H. Friend, Adv. Mater., 2017, 29, 1605987.

24 V. Gray, D. Dzebo, M. Abrahamsson, B. Albinsson and K. Moth-Poulsen, Phys. Chem. Chem. Phys., 2014, 16, 10345-10352.

25 Y. Y. Cheng, B. Fückel, T. Khoury, R. G. C. R. Clady, N. J. Ekins-Daukes, M. J. Crossley and T. W. Schmidt, J. Phys. Chem. A, 2011, 115, 1047-1053.

26 P. C. Boutin, K. P. Ghiggino, T. L. Kelly and R. P. Steer, J. Phys. Chem. Lett., 2013, 4, 4113-4118.

27 S. H. Lee, D. C. Thévenaz, C. Weder and Y. C. Simon, J. Polym. Sci., Part A: Polym. Chem., 2015, 53, 1629-1639.

28 V. Gray, K. Moth-Poulsen, B. Albinsson and M. Abrahamsson, Coord. Chem. Rev., 2018, 362, 54-71.

29 J. C. de Mello, H. F. Wittmann and R. H. Friend, Adv. Mater., 1997, 9, 230-232.

30 S. N. Sanders, E. Kumarasamy, A. B. Pun, M. T. Trinh, B. Choi, J. Xia, E. J. Taffet, J. Z. Low, J. R. Miller, X. Roy, X.-Y. Zhu, M. L. Steigerwald, M. Y. Sfeir and L. M. Campos, J. Am. Chem. Soc., 2015, 137, 8965-8972.

31 S. N. Sanders, E. Kumarasamy, A. B. Pun, M. L. Steigerwald, M. Y. Sfeir and L. M. Campos, Angew. Chem., Int. Ed., 2016, 55, 3373-3377.

32 S. N. Sanders, E. Kumarasamy, A. B. Pun, K. Appavoo, M. L. Steigerwald, L. M. Campos and M. Y. Sfeir, J. Am. Chem. Soc., 2016, 138, 7289-7297.

33 E. Kumarasamy, S. N. Sanders, A. B. Pun, S. A. Vaselabadi, J. Z. Low, M. Y. Sfeir, M. L. Steigerwald, G. E. Stein and L. M. Campos, Macromolecules, 2016, 49, 1279-1285.

34 T. N. Singh-Rachford and F. N. Castellano, J. Phys. Chem. A, 2008, 112, 3550-3556.

35 M. J. Frisch, G. W. Trucks, H. B. Schlegel, G. E. Scuseria, M. A. Robb, J. R. Cheeseman, G. Scalmani, V. Barone, B. Mennucci, G. A. Petersson, H. Nakatsuji, M. Caricato, X. Li, H. P. Hratchian, A. F. Izmaylov, J. Bloino, G. Zheng, J. L. Sonnenberg, M. Hada, M. Ehara, K. Toyota, R. Fukuda, J. Hasegawa, M. Ishida, T. Nakajima, Y. Honda, O. Kitao, H. Nakai, T. Vreven, J. A. Montgomery, J. E. Peralta, F. Ogliaro, M. Bearpark, J. J. Heyd, E. Brothers, K. N. Kudin, V. N. Staroverov, R. Kobayashi, J. Normand, K. Raghavachari, A. Rendell, J. C. Burant, S. S. Iyengar, J. Tomasi, M. Cossi, N. Rega, J. M. Millam, 
M. Klene, J. E. Knox, J. B. Cross, V. Bakken, C. Adamo, J. Jaramillo, R. Gomperts, R. E. Stratmann, O. Yazyev, A. J. Austin, R. Cammi, C. Pomelli, J. W. Ochterski, R. L. Martin, K. Morokuma, V. G. Zakrzewski, G. A. Voth, P. Salvador, J. J. Dannenberg, S. Dapprich, A. D. Daniels, O. Farkas, J. B. Foresman, J. V. Ortiz, J. Cioslowski and D. J. Fox, Gaussian 09, Revis. D.01, Gaussian, Inc., Wallingford CT, 2009.

36 N. V. Korovina, S. Das, Z. Nett, X. Feng, J. Joy, R. Haiges, A. I. Krylov, S. E. Bradforth and M. E. Thompson, J. Am. Chem. Soc., 2016, 138, 617-627.
37 A. M. Müller, Y. S. Avlasevich, W. W. Schoeller, K. Müllen and C. J. Bardeen, J. Am. Chem. Soc., 2007, 129, 14240-14250.

38 C. E. McCusker and F. N. Castellano, Inorg. Chem., 2015, 54, 6035-6042.

39 J. Zhao, K. Xu, W. Yang, Z. Wang and F. Zhong, Chem. Soc. Rev., 2015, 44, 8904-8939.

40 Y. Y. Cheng, T. Khoury, R. G. C. R. Clady, M. J. Y. Tayebjee, N. J. Ekins-Daukes, M. J. Crossley and T. W. Schmidt, Phys. Chem. Chem. Phys., 2010, 12, 66-71.

41 A. Haefele, J. Blumhoff, R. S. Khnayzer and F. N. Castellano, J. Phys. Chem. Lett., 2012, 3, 299-303. 